\title{
Traducir para el paciente: acercamiento y adaptación como modalidad de traducción
}

\author{
Translating for patients: approaching and adapting \\ as a translating method \\ Íngrid Cobos López
}

Universidad de Córdoba. icobos@uco.es

Recibido: 30.04.2019. Aceptado: 14.07.2019

Resumen: Es ingente la cantidad de publicaciones de carácter científico relacionadas con el cáncer que muestran los avances en torno a su prevención, detección precoz, sintomatología, tratamiento y otras cuestiones derivadas de un proceso cancerígeno. La mayoría de estas publicaciones, no obstante, se encuentran en inglés, lengua vehicular fundamental, y en todas ellas se ponen de manifiesto aspectos en torno a un complejo tecnolecto, de difícil comprensión por parte del enfermo. Con el objetivo de transmitir dicho conocimiento científico de una forma clara y concisa para el paciente, en el presente trabajo, propondremos unas directrices para la correcta traducción y adaptación de dichos textos de una manera directa y más cercana.

Palabras clave: discurso especializado o tecnolecto; traducción y adaptación al paciente; traducción biosanitaria; Plain English Campaign.

\begin{abstract}
There are a lot of scientific publications related to cancer regarding the latest advances about its prevention, early detection, symptomatology, treatment and other issues derived from a carcinogenic process. However, most of these papers are in English, the basic vehicular language, and we can find its complex technolect in all of them; a technolect which is quite difficult to understand by the patient. With the aim of informing about this scientific knowledge in a clear and concise way for the patient, in the present work, we will propose some guidelines for the correct translation and adaptation of the mentioned texts in a direct and closer way to the patient.
\end{abstract}

Keywords: specialised discourse or technolect; translation and adaptation for the patient; biomedical translation; Plain English Campaign.

》) Cobos López, Íngrid. 2019. "Traducir para el paciente: acercamiento y adaptación como modalidad de traducción”. Quaderns de Filologia: Estudis Lingüístics XXIV: 211-228. doi: 10.7203/QF.24.16307 



\section{Introducción}

La investigación en el campo de la traducción biosanitaria ha captado la atención de numerosos autores en las últimas décadas debido a que se trata de una herramienta para la transmisión del conocimiento científico. En este sentido, Bueno García (2007), García Izquierdo (2009), Martínez López (2009, 2010) y Lázaro Gutiérrez (2014), entre otros, abordan dicho concepto y la forma de acercarse a esta materia con propuestas metodológicas, análisis terminológicos y fraseológicos para la transmisión de dicho conocimiento especializado de la manera adecuada.

Sin embargo, la forma apropiada no siempre cumple el objetivo último que tiene su transmisión, es decir, que dicha información llegue a su destinatario de forma clara y concisa ${ }^{1}$. Por todos es sabido que existen ciertas dificultades de comunicación médico-paciente (Mayor Serrano, 2016), pero que no son exclusivas de esta disciplina.

Por ello, si retomamos la idea de la transmisión del conocimiento científico en medicina, rara vez observaremos que dicho conocimiento llegue a los pacientes de forma clara, concisa y directa por parte de los especialistas. En este sentido, autores como Córdoba (1998), Gal (2005), Domínguez-Nogueira (2005), Mayor Serrano (2008, 2016), Green y Myers (2010) y Navarro (2015), entre otros, proponen una serie de actuaciones para adaptar dicho conocimiento especializado para que sea comprensible por parte de los pacientes y sus familiares.

Partiendo de las premisas expuestas por los autores anteriores y del firme convencimiento de que es necesario acercar a los pacientes toda la investigación que se está desarrollando en torno a sus enfermedades, para que así tengan a su alcance información veraz y actual, máxime en el mundo actual lleno de fake news en torno a la salud, surge el proyecto ONCOTRAD, que tiene como objetivo traducir y adaptar las investigaciones más novedosas en torno al cáncer para que los pacientes y su entorno puedan acceder a ellas.

\footnotetext{
${ }^{1}$ En ese sentido, en 1979 se inició en Reino Unido una campaña, Plain English Campaign, para simplificar el lenguaje especializado de los organismos gubernamentales y oficiales por la que consideraban que todo el mundo debería de tener acceso a una información clara y concisa. Este movimiento surgió en torno al lenguaje jurídico aunque, no obstante, ha permeabilizado otros ámbitos de conocimiento.
} 
Para ello, y dentro del marco de dicho proyecto, este trabajo tiene los siguientes objetivos:

- revisar la bibliografía existente en torno a los géneros médicos,

- revisar la bibliografía existente en torno a la comunicación con los pacientes,

- proponer una serie de técnicas/recursos para traducir y adaptar textos sobre oncología y

- traducir y adaptar un texto actual y sobre un tema relevante para la sociedad.

\section{Tipologías y géneros médicos}

Hasta finales del siglo pasado, la traducción especializada, y por ende la traducción biosanitaria ${ }^{2}$, no recibió la atención que requería en las investigaciones en Traducción e Interpretación; no por falta de interés por parte de los autores, sino por la escasa formación e inversión que recibía este campo. Sin embargo, en las últimas décadas ${ }^{3}$, son muchos los investigadores que han abordado su estudio desde distintas perspectivas (Bueno García, 2007; García Izquierdo, 2009; Martínez López, 2009, 2010; Lázaro Gutiérrez, 2014; Mayor Serrano, 2002). La variedad de situaciones comunicativas que integra este género textual y el nivel de especialización son muy extensos y variados, ya que podemos encontrar desde un artículo científico o una patente, hasta una anamnesis o un folleto informativo para pacientes. En la actualidad, el proceso de democratización del acceso a la información y la tendencia a la educación del paciente han propiciado que se produzca un fenómeno de

\footnotetext{
${ }^{2}$ Este tipo de traducción ha recibido varias denominaciones a lo largo del tiempo entre las que destacan: traducción biomédica (Vázquez, 2006), traducción médico-sanitaria (Montalt y Shuttleworth, 2012), traducción en el ámbito biosanitario (Félix y Ortega, 1998), traducción biosanitaria (García y Varela, 2009), traducción médico-farmacéutica (Van Hoof, 1979) y traducción biomédica y farmacéutica (Gouadec, 2007).

${ }^{3}$ Según Franco Aixelá (2010: 159), una vez revisada la bibliografía especializada en torno a la traducción médica recogida en BITRA, "Se trata de un campo que es objeto de un tratamiento sistemático desde hace apenas unos 20 años, con un repentino e intense crecimiento paralelo a la creación de los estudios de traducción autónomos y modernos".
} 
reformulación del conocimiento médico por parte de los especialistas ${ }^{4}$ (Muñoz-Miquel, 2016). Este fenómeno ha hecho que los modos de comunicación de los especialistas y, por ende, los métodos de traducción de los traductores médicos se modifiquen con el objetivo de transmitir dicha información de la forma más clara y precisa al paciente y su entorno. No obstante, antes de introducirnos en el proceso que ha sufrido ese cambio en la comunicación con el paciente, vamos a estudiar las clasificaciones textuales de los géneros objeto de estudio.

\subsection{Clasificaciones de los textos médicos}

Existen dos vertientes principales que han pretendido definir y explicar los textos médicos: la de las tipologías textuales y la de los géneros textuales ${ }^{5}$. Desde el punto de vista de las tipologías textuales, mostraremos la de Löning (1981), que fue la primera en realizar una clasificación de los textos médicos. En su clasificación, establece cuatro niveles principales de comunicación: oral, escrito, teórico y práctico, y a su vez, los subdivide en función del grado de competencia del emisor/receptor y la función del texto. Lo que más interesa a este estudio es que la autora se basa en el grado de competencia de los participantes y la función comunicativa, aspectos que definen nuestro proyecto, y por tanto, pone de manifiesto que este tipo de conocimiento, en los textos objeto de estudio, se transmite de y para los especialistas, dejando de lado a los pacientes.

\footnotetext{
${ }^{4}$ Una clara muestra de lo anterior la encontramos en los resúmenes obtenidos de artículos científicos que se publican para pacientes en la revista Annals of Internal Medicine. Disponible en: http://annals.org/SS/PatientInformation.aspx [Acceso 18/02/2019].

${ }^{5}$ A caballo entre las tipologías y los géneros textuales nos encontramos las clasificaciones de Pilegard (1997), quien ofrece una propuesta sobre tipos textuales médicos y géneros textuales médicos:

En cuanto a los tipos textuales, indica lo siguiente (1997: 159):

A: el contenido (textos de medicina general o medicina especializada) y su grado de tecnicidad (textos más o menos técnicos);

B: el canal de comunicación (oral, escrito) y

C: el grado de formalidad o tenor (5 tipos textuales: textbooks, journal articles, popular medicine, doctor-patient language , journal articles, popular medicine, doctor-patient language y manuals.

En cuanto al concepto de género, elabora un listado de los géneros médicos tradicionales (1997: 163): case report, disease review, dissertation, medical textbook, speech/ printed version of speeches.
} 


\begin{tabular}{|c|c|}
\hline Nivel teórico-escrito & Nivel práctico-escrito \\
\hline $\begin{array}{l}\text { 1.1 Participantes en la comunicación: } \\
\text { especialista-especialista } \\
\text { 2. Función: transmisión de conoci- } \\
\text { mientos especializados de actualidad } \\
\text { 3. Estilo: CIENTÍFICO } \\
\text { 4. Ejemplos: publicaciones, informes } \\
\text { 2.1 Participantes en la comunicación: } \\
\text { especialista-semiespecialista } \\
\text { 2. Función: transmisión de fundamen- } \\
\text { tos } \\
\text { 3. Estilo: DIDÁCTICO } \\
\text { 4. Ejemplos: manuales, libros de texto, } \\
\text { monografías } \\
\text { 3.1 Participantes en la comunicación: } \\
\text { especialista-lego } \\
\text { 2. Función: explicar, aconsejar } \\
\text { 3. Estilo: EXPLICATIVO } \\
\text { 4. Ejemplos: libros y folletos } \\
\text { 4.1 Participantes en la comunicación: } \\
\text { lego-lego } \\
\text { 2. Función: despertar el interés de la } \\
\text { opinión pública } \\
\text { 3. Estilo: DIVULGATIVO } \\
\text { 4. Ejemplos: artículos de periódico, } \\
\text { revistas de medicina }\end{array}$ & $\begin{array}{l}\text { 1.1 Participantes en la comunicación: } \\
\text { especialista-especialista } \\
\text { 2. Función: información sobre datos } \\
\text { 3. Estilo: INFORMATIVO } \\
\text { 4. Ejemplos: informes clínicos, } \\
\text { hojas de intervención quirúrgica, } \\
\text { protocolos, informes médicos } \\
\text { 2.1 Participantes en la comunicación: } \\
\text { especialista-semiespecialista } \\
\text { 2. Función: proporcionar instruccio- } \\
\text { nes de trabajo } \\
\text { 3. Estilo: DIRECTIVO } \\
\text { 4. Ejemplos: hojas de tratamiento } \\
\text { 3.1 Participantes en la comunicación: } \\
\text { especialista-lego } \\
\text { 2. Función: proporcionar indicacio- } \\
\text { nes prácticas al paciente } \\
\text { 3. Estilo: INSTRUCTIVO } \\
\text { 4. Ejemplos: dietas, prescripciones }\end{array}$ \\
\hline
\end{tabular}

Fuente: Löning (1981: 83 y ss.)

Más adelante, y dentro del mismo ámbito, Mayor Serrano (2002), basándose en las aportaciones de Isenberg (1987), Gvenzadse (1983), Gläser (1990) y Göpferich (1995a), elabora una tipología textual de orientación pragmática en la que podemos observar que los textos obje-to de estudio de nuestro proyecto pertenecen a los textos orientados al avance de la ciencia, y por tanto de la comunicación entre especialistas, y nuestro objetivo es convertirlos, con la traducción y adaptación, en textos divulgativos; lo que en dicha clasificación serían los resúmenes y recensiones: 


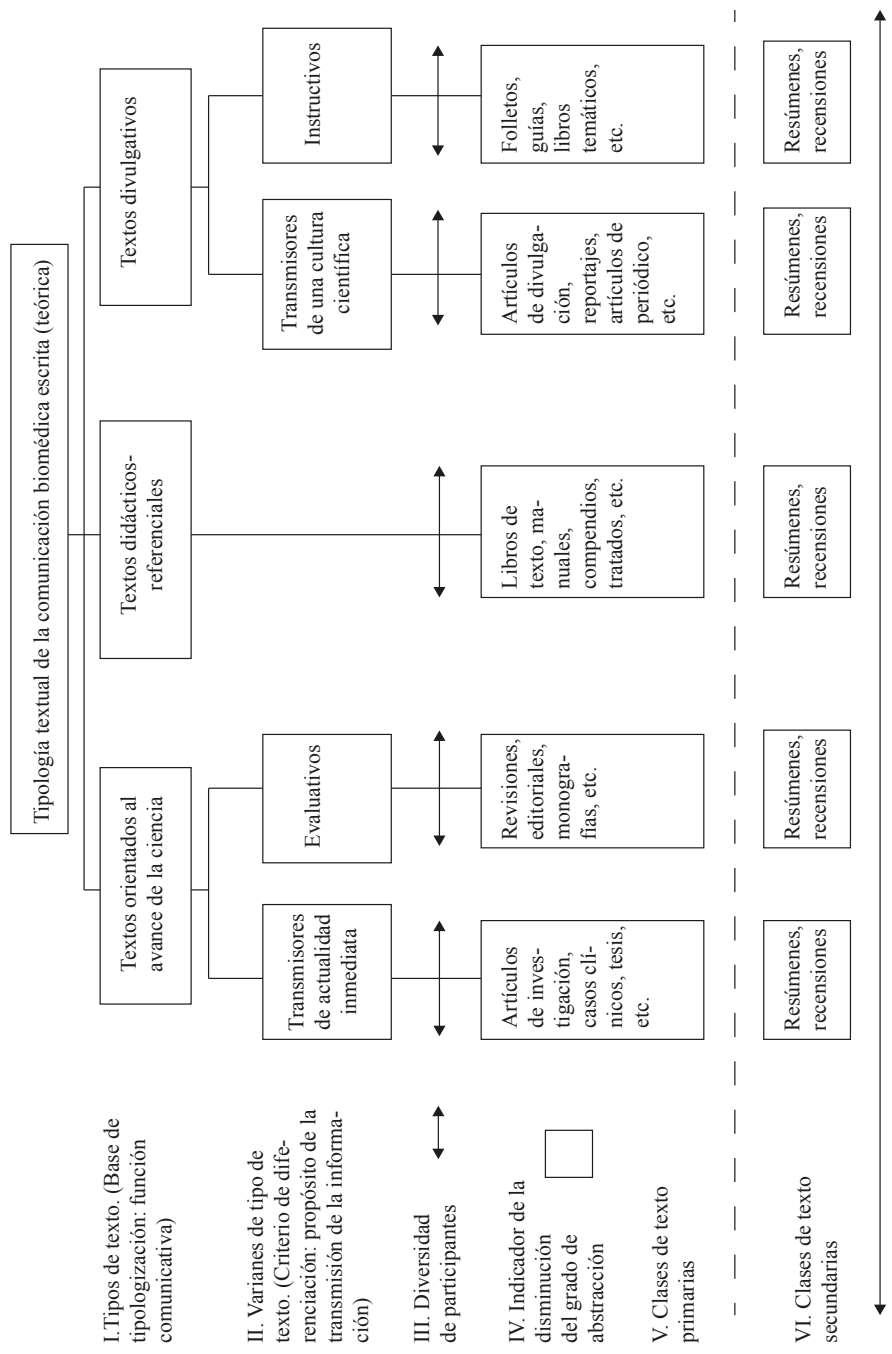

Fuente: Mayor Serrano (2002: 132)

Si bien ambas clasificaciones de las tipologías textuales nos han servido para situar nuestros textos en el contexto actual, nos basamos en la clasificación realizada por García Izquierdo (2009) por tratarse de la primera obra que analiza los géneros de divulgación médica, objeto de estudio del presente trabajo, y sobre todo, por centrarse en la "información para 
pacientes". Dicho estudio surge en el seno del equipo GENTT, del que igualmente hemos obtenido información relevante para nuestro trabajo. Dicha clasificación es mucho más amplia y descriptiva, por lo que aquí reproduciremos parcialmente la parte relativa a los documentos de información para pacientes ${ }^{6}$ :

Medical

- Clinical

- $(\ldots)$

- Patient information leaflet (PIL) / Patient package insert / Information leaflet / Consumer medicine information

- $(\ldots)$

- Popularizing

- $(\ldots)$

- Patient Information Leaflet / US Package Insert

- Medical brochure

- Popularizing guide

- Annual report

- Popularizing book

- Fact Sheet for Patients (FSP) / Patient leaflet / Patient information brochure / Patient brochure

- Summary for patients

- Tutorial

- Metagenres (...)

- Pedagogical (...)

- Advertising

- Anunci per a pacients

- Anunci per a professionals

- Advertising article

- Advertising leaflet

- Infomercial / Advertorial / Journalistic report

- (..)

- Research

- Review article

- (..)

Fuente: García Izquierdo (2009) actualizado a 2019

\footnotetext{
${ }^{6}$ Herramienta diseñada por el grupo de investigación GENTT (Géneros Textuales para la Traducción) de la Universitat Jaume I.
} 
En dicha clasificación, podemos observar que los documentos informativos para pacientes se encuentran bajo los géneros clinical, popularizing, advertising y hemos mantenido el de research puesto que la información más actual sobre medicina aparece en dichos documentos, textos a los que pretendemos que puedan acceder los pacientes y sus familiares. En esta última, observamos que hay más variedad de textos médicos para pacientes que en las de las tipologías textuales y que, aunque los artículos científicos no se encuentren en los textos para pacientes, si son un género textual identificado y que pretendemos adaptar para facilitar su accesibilidad.

\section{Traducción y adaptación para pacientes y familiares}

Encontramos hoy día un gran número de publicaciones que tienen como objetivo estudiar los elementos que contribuyen a la transmisión del conocimiento especializado a un público lego ${ }^{7}$, y la mayoría de ellas se centran en procesos de desterminologización o creación de folletos informativos para pacientes; aspectos que no están relacionados necesariamente con la traducción. Sin embargo, nuestro objetivo es acercar a los pacientes y su entorno los artículos científicos que se publican en la actualidad sobre sus patologías, en concreto la del cáncer, y hacerlo de una forma comprensible y amigable tratando de acompañar al paciente en un momento crítico en su vida.

Para ello, partiendo del concepto de desterminologización (Campos, $2008)^{8}$, vinculado a la accesibilidad del conocimiento y a la transmisión del saber científico, y usando como guía las recomendaciones de Mayor Serrano $(2007,2016)$, propondremos una serie de pautas para la traducción y adaptación de artículos científicos de actualidad para pacientes y su entorno.

\footnotetext{
${ }^{7}$ Córdoba (1998), Gal (2005), Domínguez-Nogueira (2005), Mayor Serrano (2008, 2016), Green y Myers (2010) y Navarro (2015).

${ }^{8}$ Para la autora, (2008: 49) "la desterminologización es un fenómeno formal, comunicativo y cognitivo que se manifiesta a través de una serie de procedimientos relacionados con el tratamiento de las unidades léxicas especializadas y centrados en garantizar la accesibilidad de un texto especializado a unos destinatarios no expertos." Disponible en: http://repositori.uji.es/xmlui/bitstream/handle/10234/78053/forum_2008_26. pdf? sequence=1 [Fecha de consulta: 10 de abril de 2019].
} 


\subsection{Texto objeto de estudio: Review article}

Antes de adentrarnos en las recomendaciones que nos sugiere Mayor Serrano $(2007,2016)$, analizaremos el texto objeto de estudio: el artículo de investigación. En nuestro caso, se trata de un Review article, es decir, un documento en el que un experto, grosso modo, presenta y resume la bibliografía existente en torno a un tema y aporta su visión general sobre el mismo. Dicho artículo de revisión tiene la misma estructura de un artículo de investigación (research article). A continuación, se presenta la estructura de este género textual según el ejemplo de bloques, secciones y movimientos en el artículo de investigación de Mayor Serrano (2002: 151):

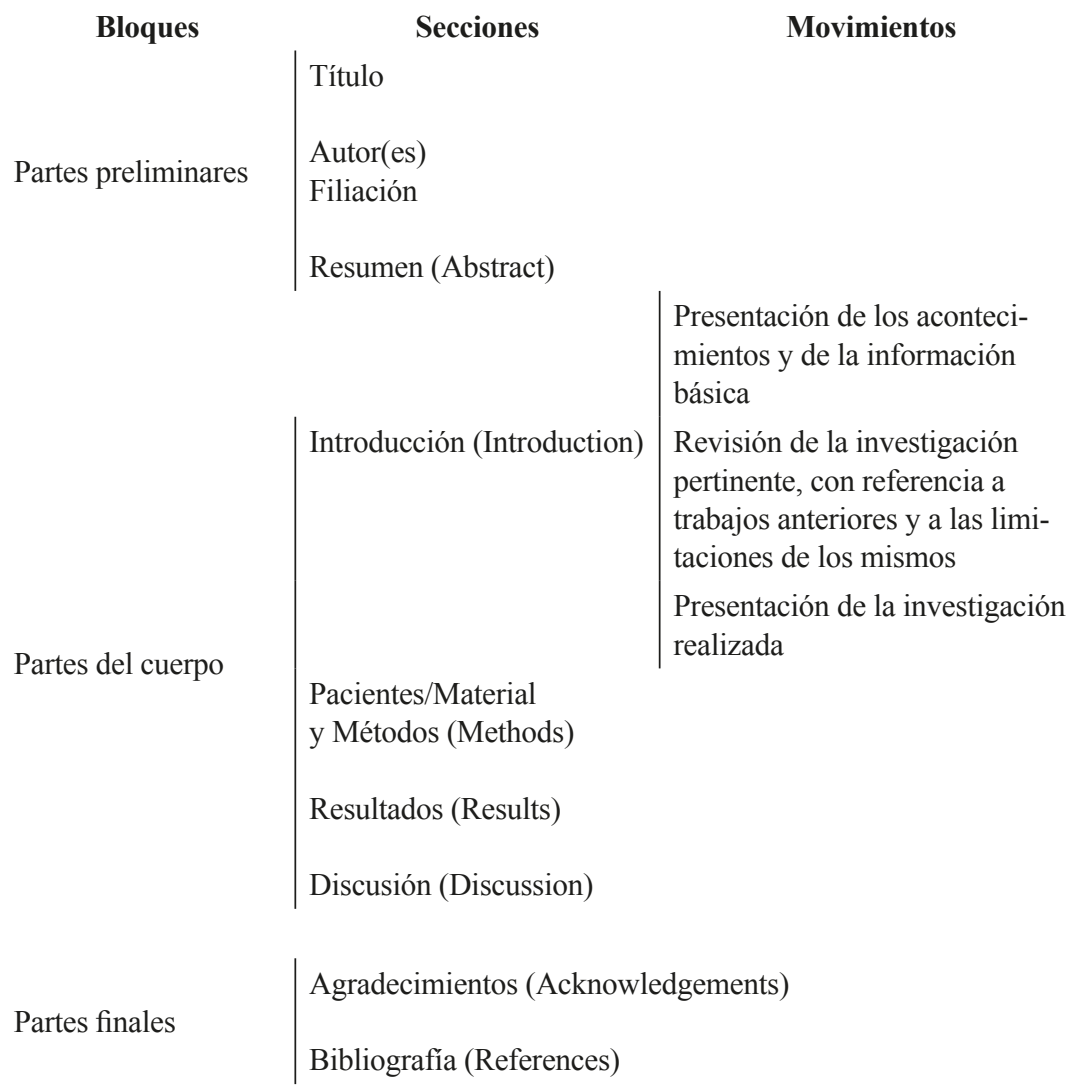


En él se presenta la macroestructura del texto y el contenido que debe de aparecer bajo cada epígrafe que, tal y como podemos observar, se trata de una estructura analítica totalmente ajena a un no experto. En primer lugar, nos encontramos con lo que Mayor Serrano (2002) denomina partes preliminares; en ellas se identifica el texto, el autor y se presenta el resumen que sintetiza el contenido del artículo. Este apartado es fundamental, puesto que en el resumen encontraremos la información más relevante a tener en cuenta en nuestra traducción/ adaptación. A continuación, se plantea la investigación en sí divida en apartados claramente diferenciados, a saber, la introducción, en la que se exponen los antecedentes y se revisa la bibliografía más relevante sobre el tema, se describe la hipótesis de estudio y se definen los objetivos; asimismo, se plantean los métodos de estudio o la metodología de investigación que se va a llevar a cabo y se indican los materiales/ pacientes que forman parte de la misma. Posteriormente, se proponen los resultados y se formula la discusión, parte esencial en un artículo científico. Por último, encontraremos los agradecimientos y la bibliografía. Este formato sería lo primero que tenemos que modificar para hacerlo accesible, ya que un lego no está familiarizado con él. Con respecto al lenguaje, cabe mencionar que es altamente especializado de y para especialistas en la materia, tal y como afirman autores como Navarro (2015), Mayor Serrano (2002, 2016), Green (2013), Martínez López (2010) o Muñoz-Miquel (2016) entre otros.

\subsection{Pautas para la traducción y adaptación a un documento nuevo para pacientes.}

Una vez revisada la bibliografía existente sobre la comunicación entre médicos y pacientes, observamos, como bien indica Novo et alii (2012: 118) lo siguiente:

La jerga médica, (...), provoca confusión y distanciamiento en los pacientes (...) por lo que debemos buscar un lenguaje que, (...) logre una comprensión aproximada de lo que estamos expresando.

Para solventar los problemas de dicha comunicación, Arana Amurrio (2014b), Mayor Serrano (2007, 2016) y Navarro (2015), entre otros, sugieren que se profundice en el estudio del lenguaje y las técnicas de comunicación en las facultades de medicina. No obstante, mientras esto 
sucede, estos mismos autores proponen distintos sistemas de adecuación de dicha comunicación a los pacientes. Entre los más estudiados se encuentran los relacionados con los folletos de salud. Sin embargo, en los trabajos de Green (2013, 2015) y en los de Mayor Serrano (2016), hemos descubierto una solución innovadora y que, gracias a las peculiaridades expresivas de la misma, implica de manera directa al lector, por lo que tiene una gran fuerza comunicadora. Nos referimos al cómic ${ }^{9}$.

En su estudio, Mayor Serrano (2016) lo presenta como un recurso didáctico para los estudiantes de medicina y que también podría usarse para transmitir el conocimiento a los pacientes con los que $\operatorname{tratan}^{10}$. Según la autora, (2016: 20) "Además de un lenguaje, el cómic es un magnífico medio de comunicación que goza de sus propios formatos de publicación", que Jiménez Varea (2006) y Gómez Salamanca (2013) los clasifican en formatos de inserción en prensa y propios ${ }^{11}$. Para la autora, sin embargo, únicamente son interesantes para el contexto médico y sanitario la historieta y la novela gráfica. Para ella, los objetivos que persiguen estas viñetas son (2016: 22-23):

- Contribuir a la mejora de la calidad de vida de los ciudadanos $(\ldots)$.

\footnotetext{
${ }^{9}$ Dentro de nuestro campo de investigación, Altarriba (2011: 9) lo define de la siguiente manera: "(...) nos encontramos ante una forma de expresión específica, un medio de comunicación perfectamente diferenciado, como el cine, la pintura o la literatura. Dentro de él existen -al igual que en el cine, la pintura o la literatura-géneros, subgéneros, registros, tonos, estilos... Y así tenemos historietas de humor, eróticas, sentimentales, de aventuras, de terror, de fantasía... Pero también poéticas, históricas filosóficas, periodísticas, biográficas, autobiográficas, experimentales o, simplemente inclasificables. Todas ellas están contadas siguiendo un código, una gramática pictográfica, una retórica escripto-icónica, un combinado léxico-gráfico, que las dota de originalidad al tiempo que las hace legibles".

${ }^{10}$ Esto mismo se ve apoyado por la tesis de McNicol (2014: 50) quien los prefiere a los folletos para pacientes debido a que los "health information comics have the potential to do much more than simply convey facts about an illness; they can also support patients in dealing with the social and psychological aspects of a condition".

${ }^{11}$ Jiménez Varea (2006: 198 y ss.) y Gómez Salamanca (2013: 243 y ss.) los clasifican de la siguiente manera:

- Formatos de inserción en prensa: humor gráfico o viñeta individual; tira cómica; tira, página y suplemento dominical.

- Formatos propios: cuaderno de historietas; comic book; prestige; historieta, novela gráfica, manga; webcómic.
} 
- Dar recomendaciones para la prevención de enfermedades o situaciones de riesgo (...).

- Informar sobre enfermedades (...).

- Reforzar la información verbal proporcionada a los pacientes y cuidadores (...).

- Favorecer la comprensión de una enfermedad por parte del paciente $(\ldots)$.

Para poder lograr estos objetivos a través de un cómic, el proceso de traducción y adaptación ha de pasar por distintas fases, a saber (2016):

- Un proceso de desterminologización, en el que se adapte el discurso al destinatario.

- Estrategias destinadas a facilitar la recepción por parte del paciente: explicación, definición, sinonimia y otras de base analógica.

A continuación, y basándonos en lo expuesto en las páginas precedentes, procederemos a analizar el artículo de revisión Consumption of Sugars, Sugary Foods and Sugary Beverages in Relation to Cancer Risk: A systematic Review of Longitudinal Studies ${ }^{12}$ que pretende comprobar si el consumo de azúcares en sus distintas formas conlleva algún tipo de riesgo de padecer cáncer. El artículo está publicado en la revista Annual Review y es de 2018, es decir, de actualidad.

Si bien por cuestiones de espacio no procedería añadir aquí el artículo de revisión completo, consideramos pertinente ofrecer al lector el resumen de dicho trabajo, que nos situará de manera conveniente para llevar a cabo nuestro estudio:

\begin{abstract}
High sugar intake may increase cancer risk by promoting insulin-glucose dysregulation, oxidative stress, inflammation, and body adiposity, but epidemiologic evidence is unclear. Associations between dietary sugars and lifestyle-related cancer risk from longitudinal studies were evaluated. We systematically searched PubMed, Embase, and CINAHL and identified 37 prospective cohort studies (1990-2017) reporting multivariable adjusted risk estimates for dietary sugars in relation to
\end{abstract}

\footnotetext{
${ }^{12}$ Disponible previo registro en www.annualreviews.org. N. Makarem et alii (2018).
} 
cancer. Of 15 and 14 studies on total sugar and sucrose respectively, 11 reported a null association in relation to cancer. Of 14 studies on fructose, 8 reported null associations, and 2 reported protective and 4 reported detrimental associations. In two of five studies on added sugars, a $60-95 \%$ increased cancer risk was observed with higher intakes. In 8 of 15 studies on sugary foods and beverages, a 23-200\% higher cancer risk was observed with higher sugary beverage consumption. In conclusion, most studies were indicative of a null association, but suggestive detrimental associations were reported for added sugars and sugary beverages.

KEYWORDS: cancer risk; prospective studies; sugars; sugary foods and beverages; systematic review ${ }^{13}$

Con respecto al proceso que hemos seguido para convertir el citado artículo en un documento accesible y adecuado para su difusión, hemos de decir que, por cuestiones de espacio, únicamente mencionaremos brevemente aquí cuáles han sido las fases por las que ha pasado. En primer lugar, se han traducido las 23 páginas que conforman el artículo. Posteriormente, hemos seleccionado la información significativa y útil para el público no experto con ayuda de un oncólogo y hemos seguido con el proceso de desterminologización y adaptación. En esta fase, que ha sido la que nos ha llevado más tiempo, nos planteamos el objetivo de dar respuesta a la pregunta que planteaba el propio artículo, de una manera clara y sencilla. Para ello, hemos extraído la información más relevante centrándonos en la introducción, los resultados y la discusión, puesto que la metodología y el análisis de los materiales y pacientes no era ni adecuado, ni relevante para el objetivo principal de este trabajo. Una vez seleccionada la información que debíamos transmitir al paciente, comenzamos con el proceso de desterminologización y adaptación siguiendo las estrategias presentadas por Mayor Serrano (2016). A continuación, creamos un cómic utilizando la herramienta web Pixton $^{14}$, puesto que consideramos que es la más intuitiva para crear un comic desde cero sin tener conocimientos previos. Para su elaboración, pensamos en un entorno amigable para el paciente y un contexto en el que se pudiera sentir identificado. Al tratarse de un estudio sobre el consumo de azúcar y otros edulcorantes en el que se mencionaban

\footnotetext{
${ }^{13}$ Fuente: https://www.ncbi.nlm.nih.gov/pubmed/29801420 [Acceso: 14/04/2019].

${ }^{14}$ Disponible en www.pixton.com.
} 
las bebidas carbonatadas y otros alimentos muy habituales en la dieta diaria de cualquier persona, utilizamos un restaurante como escenario y una conversación entre amigos sobre el tema. Así, de un modo sencillo y muy cercano, presentábamos la información destacada del artículo con un lenguaje próximo y asequible para cualquier lego en el tema.

A continuación se presenta el cómic resultante del presente trabajo:
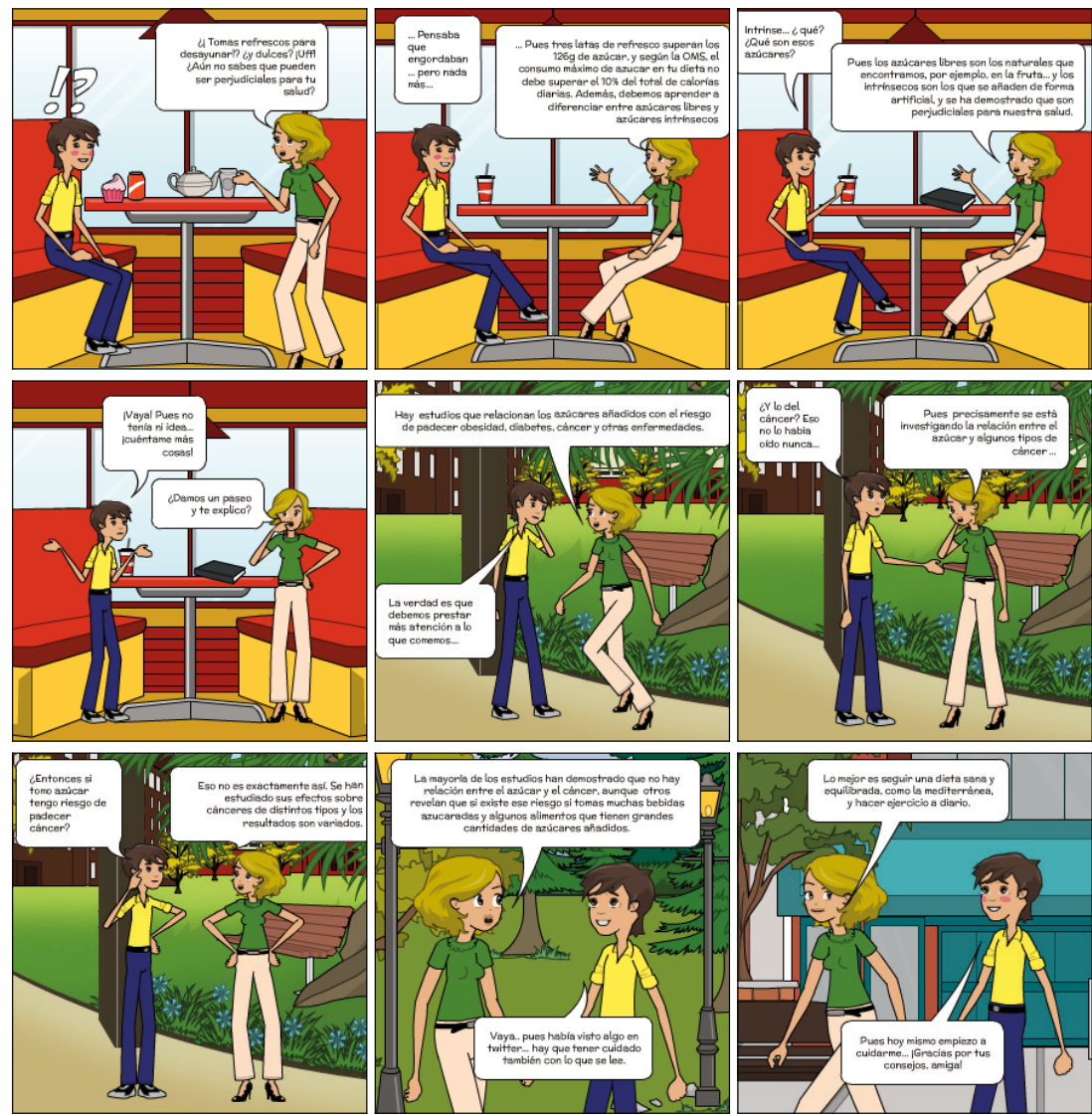


\section{Conclusiones}

El análisis efectuado en los epígrafes precedentes nos lleva a las siguientes conclusiones:

1. La traducción de textos biosanitarios es necesaria en todos sus niveles y grados; no cabe duda de que, junto a la traducción de artículos científicos, con alta densidad léxica, que permiten a los especialistas avanzar en el conocimiento científico, es también necesario un tipo de traducción dirigida hacia el paciente.

2. La traducción dirigida al paciente no puede contener la misma densidad léxica que otra tipología de textos, dado que, en definitiva, el producto de la traducción no está destinada a especialistas.

3. Por ello, también en el ámbito de la traducción biosanitaria, y teniendo en cuenta la función y el destinatario de dicha traducción, es posible un tipo de traducción que podríamos tipificar como "traducción social", en tanto que va dirigida a la ciudadanía, y a ayudarles en la gestión y la superación de su enfermedad.

4. Teniendo, pues, en cuenta, que la traducción del texto y su adaptación puede ser recibida por un amplio espectro social, de diferentes culturas, creencias y formación académica, entendemos que la traducción, unida a un proceso de transducción, es decir, presentada con otros códigos semióticos, puede ser mucho más efectiva.

5. En este sentido, el cómic se configura como el medio ideal para realizar este tipo de traducciones, pues en él se aúna la transmisión de la información principal junto a la visualización y efectividad que puede producir en quien recibe dicha traducción.

\section{Bibliografía}

Arana Amurrio, José Ignacio. 2014b. Lenguaje y labor asistencial. En Gutiérrez Rodilla, Bertha \& Navarro, Fernando (ed./coord.) La importancia del lenguaje en el entorno biosanitario. Barcelona: Fundación Dr. Antonio Esteve, 1-8. http://www.esteve.org/publicaciones/monografias/ [Acceso: 28/03/2019]

Bueno García, Antonio. 2007. Nuevas iniciativas en torno a la formación e investigación en traducción biosanitaria.Panace@: Revista de Medicina, Lenguaje y Traducción 8: 225-229. 
Campos Andrés, Olga. 2013. Procedimientos de desterminologización: traducción y redacción de guías para pacientes.Panace@: Revista de Medicina, Lenguaje y Traducción 14: 48-52. http://www.tremedica.org/ panacea/IndiceGeneral/n37-tradyterm-OCamposAndres.pdf. [Acceso: $15 / 03 / 2019]$

Córdoba García, Rodrigo. 1998. Elaboración de folletos educativos dirigidos a pacientes. Barcelona: PAPPS-semFYC. http://www.almirall.es/guies/ educ.html. [Acceso: 02/02/2019]

Franco Aixelá, Javier. 2010. Una revisión de la bibliografía sobre traducción e interpretación médica recogida en BITRA (Bibliografía de Interpretación y Traducción).Panace@: Revista de Medicina, Lenguajey Traducción 11: 151-160.

Gal. 2005. Gal I, Prigat A. Why organizations continue to create patient information leaflets with readability and usability problems: an exploratory study. Health Education Research. Theory and Practice 20: 485-93.

García Izquierdo, Isabel. 2009. Divulgación médica y traducción. El género información para pacientes. Berna: Peter Lang.

Gómez Salamanca, Daniel. 2013. Tebeo, cómic y novela gráfica: la influencia de la novela gráfica en la industria del comic en España (Tesis Doctoral). Barcelona: Universitat Ramon Llull [Base de datos Teseo]. http:// www.tdx.cat/handle/10803/117214

Green, Michael J. 2013. Comics and medicine: peering into the process of professional identity formation. Academic Medicine 90(6): 1-6.

Green, Michael J. 2013. Teaching with comics: a course for fourth year medical students. Journal of Medical Humanities 34: 471-6.

Green Michael J. \& Myers, Kimberly R. 2010. Graphic medicine: use of comics in medical education and patient care. BMJ 340: 574-7.

Jiménez Varea, Jesús. 2006. El contexto de la historieta: conformación, industria y relación con otros medios. Ámbitos 15: 191-209.

Lázaro Gutiérrez, Raquel. 2014. Perspectivas en la formación de posgrado en traducción biosanitaria. Skopos. Revista Internacional de Traducción e Interpretación 4: 167-185.

Löning, Petra. 1981. Zur medizinischen Fachsprache. Stilistische Gliederung und Textanalysen. Muttersprache 91: 79-92.

Makarem, Nour; Bandera, Elisa; Nicholson, Joseph M. \& Parekh, Niyati. 2018. Consumption of Sugars, Sugary Foods, and Sugary Beverages in Relation to Cancer Risk: A Systematic Review of Longitudinal Studies. Annual Review of Nutrition 38: 17-39.

Martínez López, Ana Belén. 2009. Terminología y fraseología en los documentos médico-legales (1): extracción, clasificación, análisis y traducción de unidades de naturaleza biosanitaria. Entreculturas 1: 385-408. 
Martínez López, Ana Belén. 2010. La traducción de textos médicos especializados para el ámbito editorial (inglés-español). Granada: Comares.

Mayor Serrano, Blanca. 2002. Tipología textual pragmática y didáctica de la traducción en el ámbito biomédico (Tesis Doctoral). Granada: Universidad de Granada.

Mayor Serrano, Blanca. 2007. Cómo elaborar folletos de salud destinados a los pacientes. Barcelona: Fundación Dr. Antonio Esteve.

Mayor Serrano, Blanca. 2016. El cómic como recurso didáctico en los estudios de medicina. Barcelona: Fundación Dr. Antonio Esteve.

McNicol, Sarah. 2014. Humanising illness: presenting health information in educational comics. Med Humanit 40: 49-55.

Muñoz-Miquel, Ana. 2016. La traducción médica como especialidad académica: algunos rasgos definitorios. Hermeneus 18: 235-267.

Navarro, Fernando. 2015. Los médicos, ¿se explican bien o mal? Laboratorio del lenguaje. http://medicablogs.diariomedico.com/laboratorio/2015/01/05/los-medicos-se-explican-bien-o-mal/ [Acceso: 21/01/2019]

Novo Rodríguez, José M. \& Martínez Anta, M. J. 2012. Doce más una estrategias erróneas para dar malas noticias. Galicia Clínica 73(3):117-9.

Pilegard, Morten. 1997. Translation of Medical Research Articles. En Trosborg, Anna (ed.) Text Typology and Translation. Ámsterdam/Filadelfia: John Benjamins: 159-184. 